\title{
Criterios \\ Torres de vigilancia costera y Paisaje: integración de valores culturales y naturales en el Paraje Natural de Maro-Cerro Gordo
}

Marta Chiclana Rodriguez > Silvia Fernández Cacho

Instituto Andaluz del Patrimonio Histórico

\section{Resumen}

En la actualidad, el creciente interés por la tutela del Patrimonio Histórico ha impulsado los estudios en este campo, en cuya evolución se observa la tendencia de integrar los valores culturales y naturales en la ordenación y gestión del territorio. Por otra parte, la consolidación de los Sistemas de Información Geográfica (SIG) como técnica de tratamiento cartográfico para el análisis espacial, proporciona amplias posibilidades como elemento de estudio de la realidad geográfica. En este trabajo se muestran los resultados de un ejemplo de la aplicación del análisis espacial a través de un SIG para analizar conjuntamente, desde la base de su representación cartográfica, los valores culturales y naturales en un Espacio Natural Protegido. El objetivo es realizar una propuesta de protección y valorización paisajistica, considerando como elementos clave las cuencas visuales de las torres de vigilancia costera, de los caminos que les dan acceso y del grado de antropización del medio en el entorno del espacio natural.

\section{Palabras clave}

\section{Andalucia}

Costa

Paisaje

Patrimonio cultural

Patrimonio inmueble

Patrimonio natural

Paraje Natural de Marco-Cerro Gordo Torres defensivas

\section{Patrimonio Cultural y Patrimonio Natural: una integración necesaria}

En la actual organización administrativa autonómica y estatal, la gestión del Patrimonio Histórico se realiza de forma independiente a la relativa al medio ambiente, la protección de la naturaleza y el Patrimonio Natural. Algunos de los motivos de esta separación son meramente instrumentales: es necesario sectorizar las inversiones, la toma de decisiones, la jerarquía departamental, etc. para poder organizar la complejidad de las innumerables actuaciones, programas y proyectos que se ejecutan por parte de los poderes públicos tanto a escala nacional, como regional o local. Pero podrian destacarse otros aspectos que explican esta situación:

> Diferente consideración social: En los últimos decenios el movimiento ecologista ha impulsado en toda Europa una mayor conciencia sobre la necesidad de conservar el medio ambiente y con este fin se han multiplicado las inversiones, se han promovido normas legales y se ha provisto a las administraciones medioambientales de unos recursos económicos no comparables a los destinados a la conservación del Patrimonio Histórico, cuya defensa no ha sido tan decidida ni ha calado con la misma intensidad en el conjunto de la población. Como planteaba N. Bouché en 1995 "en toda Europa occidental, los movimientos ecologistas han generado el reforzamiento de la protección de la naturaleza y la prioridad acordada sobre este aspecto del medioambiente, a veces en detrimento de la toma en consideración del patrimonio y de los paisajes construidos" (Bouché, 1995: 194), proceso que no ha tenido parangón en el campo del Patrimonio Histórico hasta el momento.

> Especialización del conocimiento: La especialización del conocimiento ha sectorizado profundamente los estudios universitarios (Rosmaninho, 2000: 8-9; Castro, 2000: 43; Zifferero, 1999: 319). La necesaria consecuencia de esta situación es la falta de consideración integral de ambos tipos de patrimonio, resultando más fácil abordar trabajos específicos en ámbitos de conocimiento concretos, que trabajar en torno a una "categoría difusa" más integradora que no es reconocida claramente por las diferentes especialidades. Sin embargo, cada vez se hace más necesaria una estrecha colaboración entre todas las disciplinas involucradas en el estudio del medio ambiente (Gestin, 1995: 96), del que el Patrimonio Histórico es un elemento integrante más, a pesar de que, desde el punto de vista administrativo, sea más sencillo elaborar propuestas de intervención sin el concurso de especialistas procedentes de tan diversas áreas de conocimiento.

> Diferentes posicionamientos epistemológicos: La sectorización de los estudios universitarios no hace sino materializar a nivel formativo las diferencias entre las concepciones del mundo de unas y otras disciplinas alineadas en torno a las ciencias humanas y a las ciencias naturales, sobre todo desde que se aceptó la incorporación del ser humano como una especie más de la cadena evolutiva y se mar- 


\section{Criterios}

Torres de vigilancia costera y Paisaje:

integración de valores

culturales y naturales en

el Paraje Natural de

Maro-Cerro Gordo

\section{Marta Chiclana Rodríguez}

Silvia Fernández Cacho
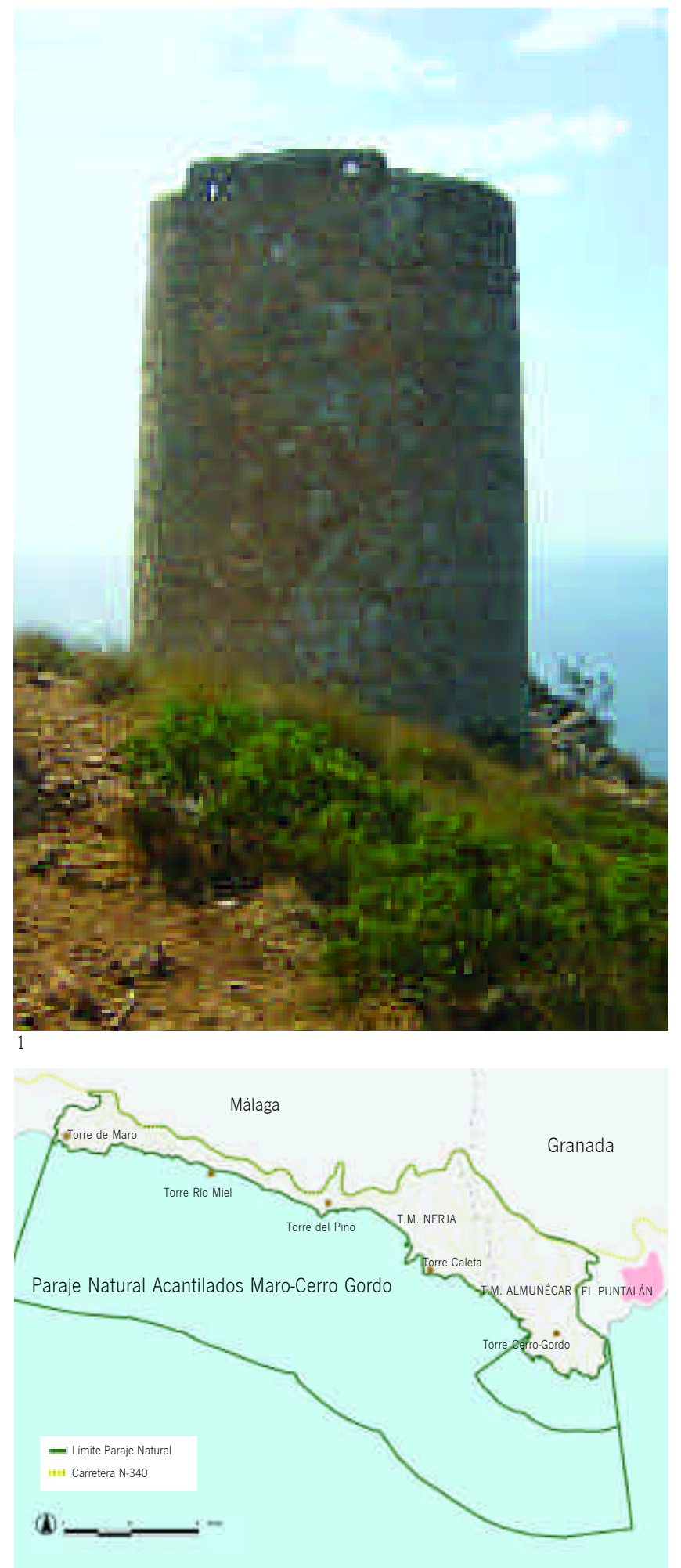

Figura 1
1. Torre Cerro-Gordo

Figura 1. Localización del Paraje Natural Maro-Cerro Gordo

2. Torre del Rio de la Miel

3. Torre Caleta

4. Torre de Maro

5. Torre del Pino caron las diferencias entre especies "sin cultura" y "especies con cultura” (Blanckaert, 1998; Kuper, 1998; Corbey, 1998).

La distinta posición epistemológica entre ambos campos de conocimiento se resume en el hecho de que para las ciencias humanas la historia, el encadenamiento de los fenómenos y la causalidad forman parte de su razonamiento explicativo, mientras que en las ciencias naturales los fenómenos pueden ser independientes unos de otros (Van der Leeuw, 1998), minimizándose la componente histórica y los procesos de cambio en muchas de sus investigaciones.

Desde la óptica del desarrollo sostenible, con su vocación de puente entre pasado y futuro (Gutiérrez-Cortines, 2002), la preservación de un tipo de patrimonio frente al descuido del otro es injustificable. El patrimonio de una comunidad debe ser conservado y transmitido, sea dicha conservación integral (reservas naturales, sitios arqueológicos, monumentos, etc.) o parcial, es decir, centrada en el mantenimiento de los factores esenciales que lo caracterizan (parques naturales, ciudades históricas, áreas de interés arqueológico, paisajes culturales, etc.). En ambos casos, sean cuales fueren los valores predominantes del lugar (culturales o naturales) el efecto de una tutela centrada en un único aspecto irá siempre en detrimento de la valoración del conjunto (Castro, 2000).

\section{El Patrimonio Arqueológico en los espacios naturales protegidos}

Un eje fundamental de las politicas medioambientales y culturales viene trazado por la conservación de aquellas entidades de carácter diverso que se han considerado como partes integrantes del patrimonio de la sociedad. La administración medioambiental ha delimitado extensas áreas territoriales en las que planifica y ordena las actividades y usos antrópicos con el fin de garantizar dicha conservación. Sin embargo, el Patrimonio Histórico en estas áreas a menudo no recibe una especial atención a pesar de que, en principio, el esfuerzo para su conservación y disfrute dentro de estos espacios es mucho menor que fuera de ellos, ya que son áreas protegidas en las que existe una regulación amplia de usos y aprovechamientos del territorio.

En la tendencia que se observa en las orientaciones europeas sobre conservación de la naturaleza hacia la integración de los aspectos culturales influye, por un lado, la carga proyectual de la planificación de los espacios protegidos y, por otro, la propia evolución del concepto de Patrimonio Histórico. El primer aspecto deriva de la necesidad de favorecer el desarrollo económico sin perjudicar el medio ambiente, es decir, haciendo una apuesta explícita por el desarrollo sostenible. Por otra parte, el Patrimonio Histórico ya no ciñe su ámbito de tutela a objetos singulares más o menos monumentales, sino que se extiende a aspectos intangibles de la cultura y a ámbitos territoriales más o menos extensos en los que sus características y valores culturales así lo hace necesario (Peano, 1999). 

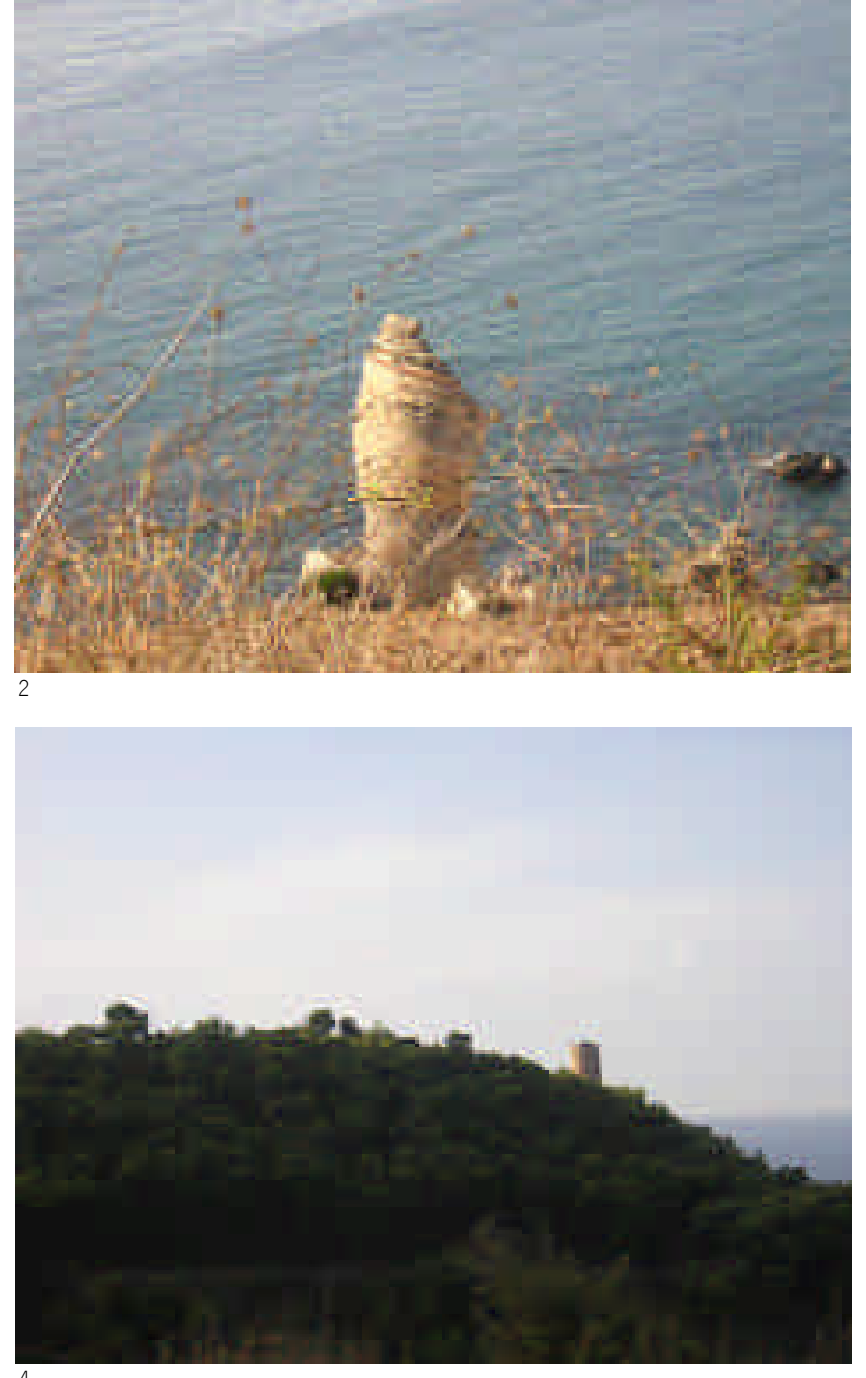

En los espacios naturales protegidos la tutela del Patrimonio Histórico podría constituir una línea prioritaria de acción conjunta de las administraciones implicadas en torno a tres principios fundamentales:

$>$ Promoción del conocimiento de los propios espacios.

$>$ Racionalización y equilibrio en las inversiones.

> Potenciación de la sinergia en las actuaciones de difusión y valorización del Patrimonio Natural y Cultural.

En efecto, el esfuerzo realizado para la protección de los espacios naturales debería contribuir a la más efectiva preservación de los bienes culturales que se sitúan en ellos y que son la expresión de la ocupación humana del medio físico y del aprovechamiento de sus recursos. En este contexto, los espacios naturales protegidos pueden ser los lugares ideales para la experimentación de políticas de tutela con objetivos comunes, sobre todo en aquellos en los que la conjunción de valores naturales y culturales es más sobresaliente (Rosmaniho, 2000).

Se trata de aunar esfuerzos y racionalizar la gestión, evitando la duplicidad y el solapamiento de los instrumentos de tutela (Nuzzo, 1999). La conservación y el uso racional de los recursos patrimoniales es un objetivo común, por lo que se debe garantizar la pervivencia para las generaciones futuras de las especies vegetales y animales, los paisajes y los elementos significativos de la actividad humana, que caracterizan un territorio concreto y que se circunscriben tanto a inmuebles 0 actividades tradicionales recientes, como también pretéritas.

El desarrollo sostenible hacia el que se dirigen todas las políticas de ordenación de los espacios protegidos a nivel regional, nacional e internacional, no puede en ningún caso lograrse si a la vez que se preserva su Patrimonio Natural, la falta de una coordinación y cola-
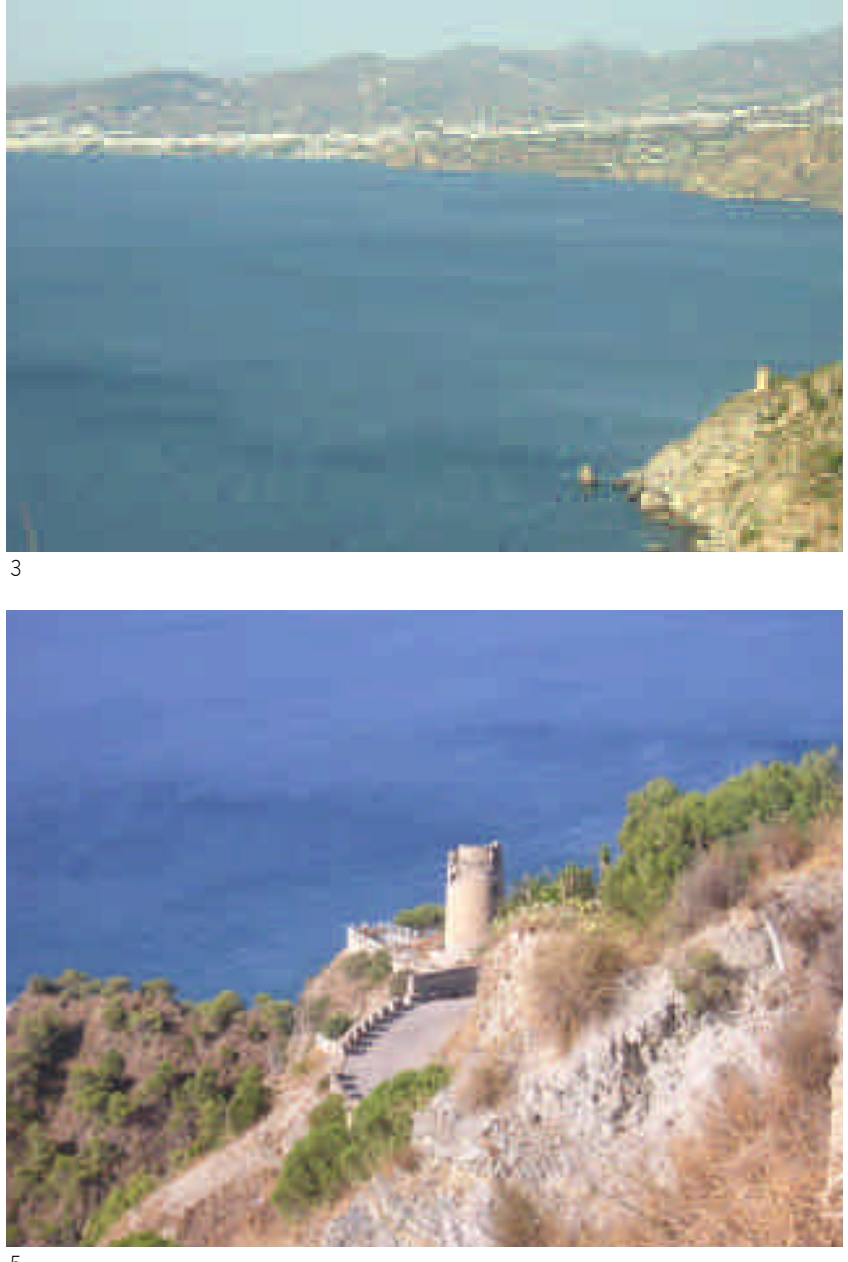

boración adecuada provoca la pérdida de su Patrimonio Histórico o, cuanto menos, su escaso uso. Ambos se consideran objeto de protección, patrimonio de la sociedad en su conjunto y la administración tiene la obligación de crear los cauces necesarios para garantizar su conservación. Como expone A. Nuzzo "...este diseño unitario, asociando el valor natural, o cuanto menos ambiental, al cultural; abriendo el camino a la integración entre todas las políticas de intervención orientadas al desarrollo sostenible; proponiendo proyectos integrados y de áreas vastas, de referencia para pactos y acuerdos territoriales constituye la única vía de salida en un recorrido que, hasta hoy, parece llevar a un callejón sin salida" (Nuzzo, 1999: 332).

\section{Propuesta de revalorización paisajística de las Torres vigía en el Paraje Natural de Maro-Cerro Gordo}

\section{El contexto}

El Paraje Natural Acantilados de Maro-Cerro Gordo está situado frente al mar de Alborán, entre las provincias de Málaga y Granada, concretamente, entre la aldea de Maro, perteneciente al término municipal de Nerja (Málaga), y el límite occidental de la costa de Granada, en la Herradura, correspondiente al municipio de Almuñécar.

El área del paraje natural queda restringida a una banda de $12 \mathrm{Km}$ de longitud perpendicular a la línea de costa, acotada en el norte por la carretera N-340 y extendiéndose hacia el sur, una milla náutica en el interior del Mar de Alborán (figura 1). La superficie total protegida del espacio abarca $1.815 \mathrm{Ha}$, y se caracteriza fundamentalmente por 


\section{Criterios}

Torres de vigilancia costera y Paisaje:

integración de valores

culturales y naturales en

el Paraje Natural de

Maro-Cerro Gordo

\section{Marta Chiclana Rodríguez}

Silvia Fernández Cacho

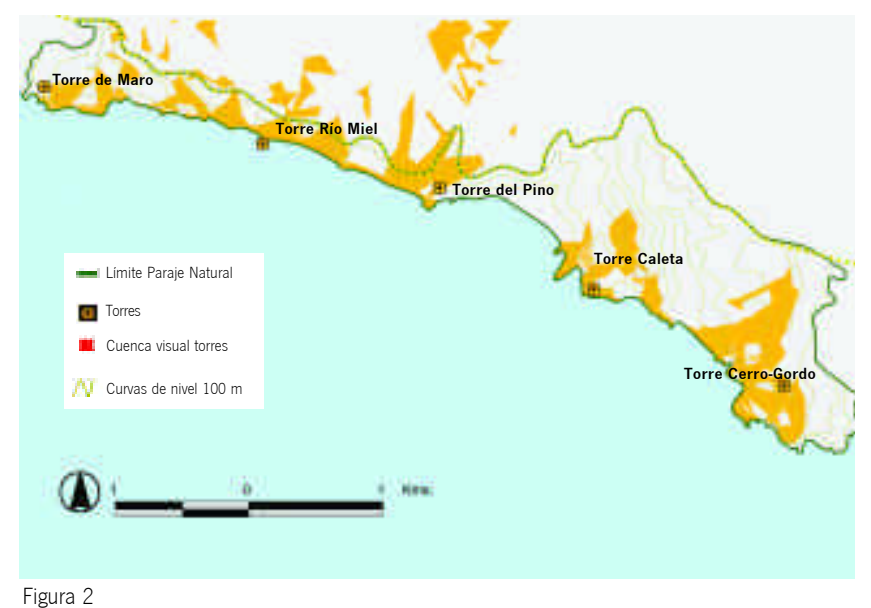

una estructura de acantilados marinos, calas y pequeñas playas que se extienden a lo largo del litoral.

Es un enclave de gran valor ambiental, por la variedad de su flora y fauna y la diversidad de los complejos ecológicos de los que forman parte, incluidos los ecosistemas terrestres y marinos. Por esta razón, la Ley 2/89, de 18 de julio del Inventario de los Espacios Naturales Protegidos de Andalucía, declara los Acantilados de Maro- Cerro Gordo como Paraje Natural, quedando incluido en la Red de Espacios Naturales Protegidos de Andalucia (RENPA).

Este paraje natural también fue propuesto en el año 1998 como Lugar de Interés Comunitario (LIC), contando con el régimen de protección de la Directiva Hábitat de la UE (Directiva 92/43 CEE) que tiene como objetivo básico el sostenimiento de la biodiversidad mediante la conservación de los hábitat naturales y de la flora y fauna silvestres. De igual modo, por la peculiaridad de sus formaciones geológicas y la gran biodiversidad que alberga en su espacio fue declarado en el año 2003 como ZEPIM (Zona Especialmente Protegida de Importancia para el Mediterráneo), en el marco del Convenio de Barcelona para la Protección del Mediterráneo.

Al margen de sus excepcionales condiciones medioambientales, los Acantilados de Maro-Cerro Gordo, constituyen un enclave estratégico desde el punto de vista del paisaje, por la singularidad de su realidad geográfica y los valores de su relevante Patrimonio Histórico. En efecto, al contrastado interés natural de este espacio protegido hay que sumarle la significativa presencia de torres de vigilancia costera, elementos que condicionan en gran medida la notoriedad de este entorno natural acentuando su riqueza cultural y su singular paisaje.

En el paraje natural de Maro-Cerro Gordo, se localizan concretamente 5 torres de vigilancia costera de los siglos XV-XVI (Torre de Maro, Torre Río de la Miel, Torre del Pino, Torre Caleta y Torre de CerroGordo) (imágenes 1-5), que forman parte de un complejo sistema de
Figura 2. Cuencas visuales de las torres

Figuras 3-26: Cuencas visuales de los 24 caminos del Paraje natural

control y comunicaciones que se extiende a lo largo de toda la costa andaluza, tanto mediterránea como atlántica.

La ubicación privilegiada de estos sitios arqueológicos, con amplias cuencas visuales en razón de su funcionalidad, los convierte en lugares apropiados desde los que observar el paisaje, promocionando el uso y disfrute del entorno natural y del Patrimonio Histórico.

En este contexto, el objetivo de este trabajo es el de realizar propuestas de protección y valorización paisajística, considerando como elementos clave las cuencas visuales de las torres de vigilancia, de los caminos que les dan acceso y del grado de antropización del medio en el entorno inmediato del paraje.

\section{La propuesta}

Para acometer esta tarea se emplean como fuentes de información cartográfica el MVA 10.000 y la ortofoto digital de Andalucía a color de 1999, producidas ambas por el Instituto de Cartografía de Andalucía.

La sensación de relieve se proporciona indirectamente mediante la información de la imagen: sombras en las laderas de pendientes más acusadas, las variaciones de tono de la cubierta vegetal, la situación, etc. En este caso, para realzar el relieve se procede a superponer la ortofoto al modelo tridimensional creado a partir de la información de relieve contenida en el MVA 10.000.

Puesto que el análisis de las cuencas visuales y el establecimiento de rutas óptimas son aspectos considerados importantes desde la óptica paisajística, la propuesta desarrollada en este trabajo tratará de recogerlos e integrarlos ajustados a un enfoque patrimonial.

Analíticamente, las cuencas visuales se obtienen a partir de las intersecciones producidas por haces de rectas con origen en la persona que observa y los elementos cartografiados, teniendo en cuenta una serie de restricciones tales como la orografía o la profundidad máxima que puede alcanzar el ojo humano entre otras.

En este trabajo se han calculado las cuencas visuales tanto desde el punto de vista de un enfoque activo como pasivo. El enfoque activo analiza el área perceptible desde un punto dado, en este caso el área visible desde las torres vigía. En el caso contrario, se han examinado las zonas desde las que dichas torres son percibidas por el público que accede al paraje natural en el que se ubican.

Para calcular las cuencas visuales de las torres se ha empleado una cobertura digital puntual referida a su emplazamiento y el modelo digital de elevaciones, generado a partir de la interpolación del modeIo 3D. Este enfoque proporciona un mapa a partir del cual se ilustran aquellas áreas que son visibles desde las torres (figura 2).

Este sencillo análisis facilita un tipo de información muy útil para el objetivo propuesto: para la revalorización de las torres vigía como 


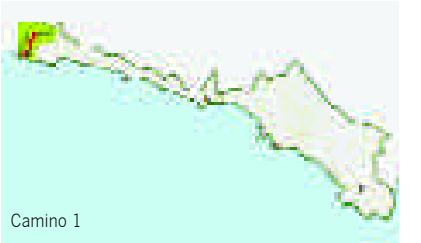

Figura 3

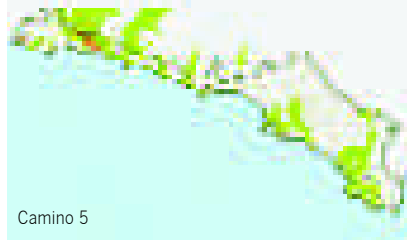

Figura 7

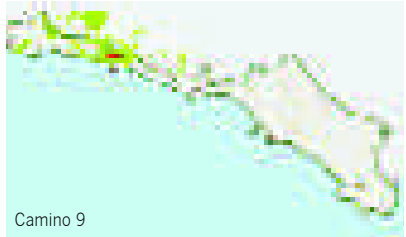

Camino

Figura 1

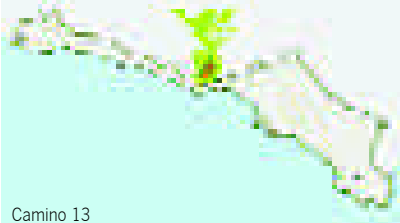

Figura 15

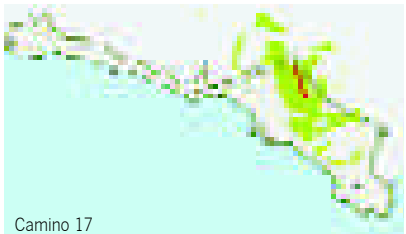

Camino 17

Figura 19

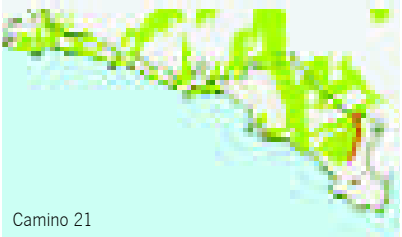

Figura 23

Limite Paraje Natural

a Torre

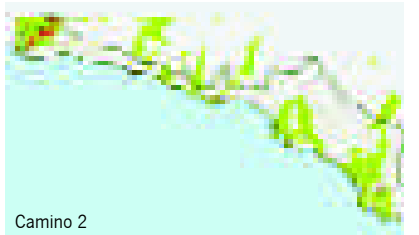

Figura 4

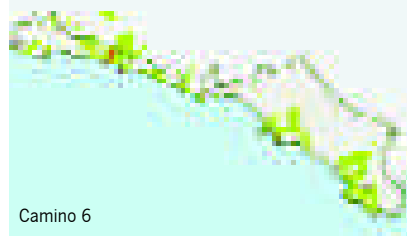

Figura 8

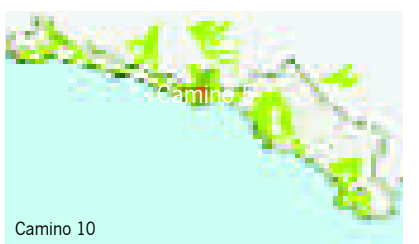

Figura 12

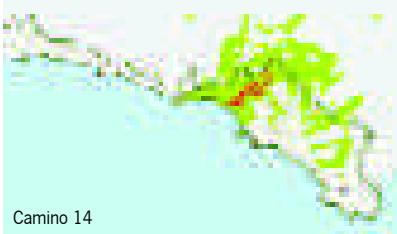

Figura 16

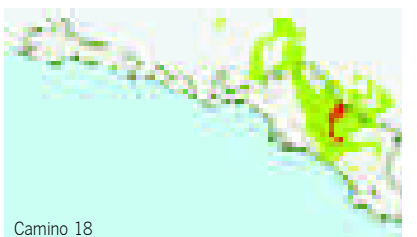

Figura 20

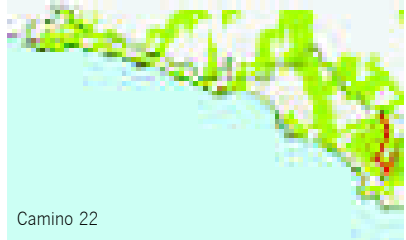

Figura 24

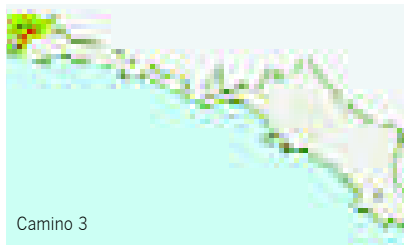

Figura 5

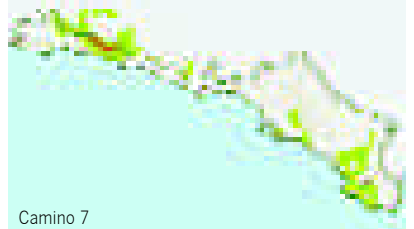

Figura 9

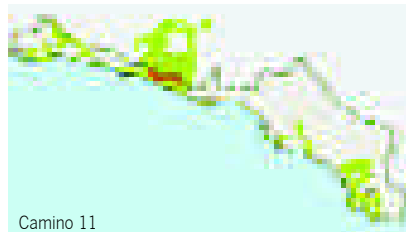

Figura 13

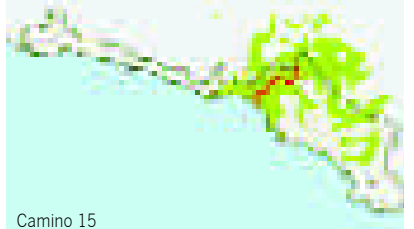

Figura 17

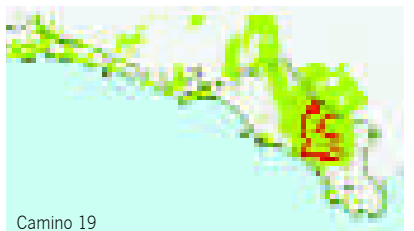

Figura 21

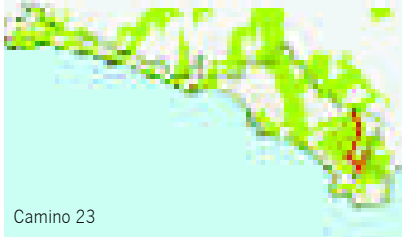

Figura 25
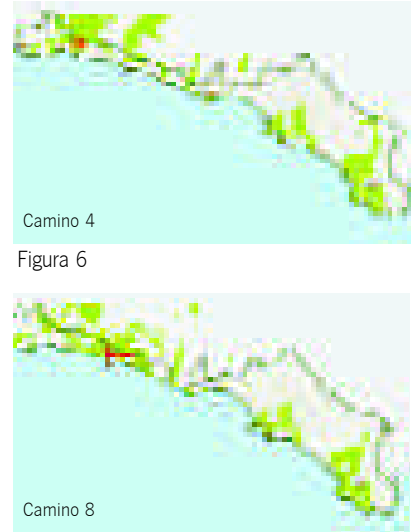

Figura 10

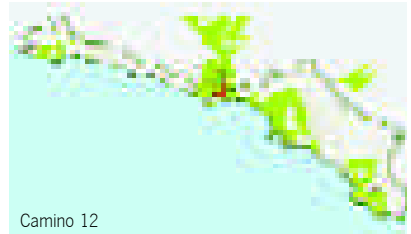

Figura 14

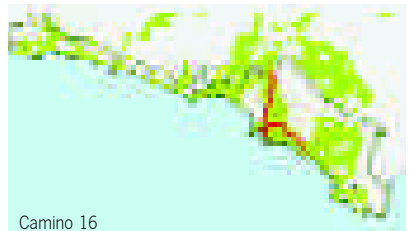

Figura 18

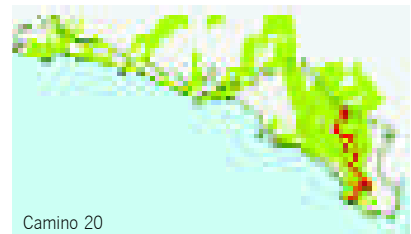

Figura 22

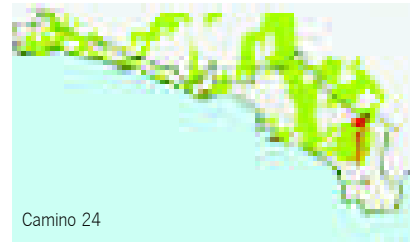

Figura 26

Caminos

N Curvas de nivel 100 m

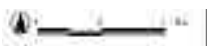

recurso patrimonial es necesario asociar a las medidas de protección concretas de las que disfrutan, otras acciones encaminadas a salvaguardar su entorno más inmediato, siendo prioritario el establecimiento de medidas de actuación en el espacio que abarca su cuenca visual. Estas acciones sobre su área de influencia más directa deben prevenir y evitar impactos paisajísticos no deseados como el deterioro o degradación de sus componentes por la presencia de procesos poco respetuosos con el medio.

También se han calculado las cuencas visuales de los caminos incluidos en el área del paraje que dan acceso a las distintas torres, con la finalidad de conocer cuales son las zonas que se perciben visualmente durante su recorrido. Ello permitirá determinar qué rutas de entre las existentes pueden potenciarse desde el punto de vista del disfrute paisajístico del paraje.

Para elegir los trayectos más singulares se tendrán en cuenta las cuencas visuales de éstos que mejor contengan las torres, además de aquellos itinerarios que se incluyan en la superficie de visibilidad de las mismas. Con este objetivo, se seleccionarán aquellos caminos que permiten encuadrar el entorno de las torres de forma adecuada (considerándose como tales las cuencas visuales de éstas), con vistas a seleccionar un determinado número de vías de acceso por su singularidad desde el punto de vista de la visibilidad y establecer medidas de protección de su espacio de visión, por los valores culturales que aportan este tipo de construcciones históricas en la zona de estudio.

A continuación se muestran en una serie de mapas, las distintas cuencas visuales de los caminos de acceso al paraje (figuras 3-26), digitalizados a partir de la ortofoto a color del año 1999. Estos tramos se presentan siguiendo el itinerario recorrido por la carretera N-340 


\section{Criterios \\ Torres de vigilancia \\ costera y Paisaje: \\ integración de valores \\ culturales y naturales en \\ el Paraje Natural de \\ Maro-Cerro Gordo \\ Marta Chiclana Rodríguez \\ Silvia Fernández Cacho}

(que limita por el norte el paraje), desde la aldea de Maro, en la provincia de Málaga (límite occidental) hasta la población de la Herradura (en el extremo suroriental), en el término municipal de Almuñécar (Granada).

Si se observan los siguientes mapas, se puede advertir a simple vista los caminos que representan un área de distribución visual más restringida, y aquellos otros que se extienden sobre una superficie más amplia. Sin embargo, los tramos que abarcan una superficie más limitada no tienen por qué resultar menos interesantes desde el punto de vista del paisaje, puesto que las extensiones comprendidas dentro de dichas áreas pueden albergar elementos destacables, y por el contrario, cuencas visuales más extensas pueden no contener unidades de especial interés.

Los criterios de selección de los caminos más idóneos para recorrer la superficie del paraje se basarán en los siguientes aspectos:

$>$ Superficie de sus cuencas visuales (tabla 1).

> Localización de torres vigía en el campo de visión durante su recorrido.

Si se relacionan las superficies que abarcan las cuencas visuales de los caminos con la proporción de su área de distribución en relación al número de torres comprendidas en éstas, se podrian seleccionar del total de caminos representados un número más reducido de éstos, con idea de distinguir aquellos recorridos más sobresalientes por la amplitud de su cuenca visual. Se trata de priorizar los itinerarios de acceso al paraje que por su campo de visibilidad delimitan superficies de clara influencia sobre el entorno de localización de las vigías, como figuras claves del espacio protegido.

Teniendo en cuenta los criterios indicados anteriormente, se decide escoger de forma prioritaria los caminos número 2, 6, 10, 14, 16 y 21 por las siguientes razones:

\begin{tabular}{|lrlr|}
\hline Tabla 1 & & & \\
Camino & Superficie & Camino & Superficie \\
Camino 1 & 340.300 & Camino 13 & 871.500 \\
Camino 2 & 4.079 .500 & Camino 14 & 3.804 .100 \\
Camino 3 & 546.400 & Camino 15 & 3.739 .400 \\
Camino 4 & 3.368 .600 & Camino 16 & 5.808 .900 \\
Camino 5 & 3.718 .800 & Camino 17 & 1.161 .100 \\
Camino 6 & 3.499 .300 & Camino 18 & 1.971 .100 \\
Camino 7 & 2.850 .700 & Camino 19 & 1.469 .200 \\
Camino 8 & 3.804 .700 & Camino 20 & 5.556 .800 \\
Camino 9 & 1.567 .000 & Camino 21 & 6.257 .600 \\
Camino 10 & 4.404 .000 & Camino 22 & 5.722 .600 \\
Camino 11 & 3.756 .600 & Camino 23 & 6.225 .900 \\
Camino 12 & 3.997 .800 & Camino 24 & 5.384 .900 \\
\hline
\end{tabular}

Tabla 1. Superficie $\left(\mathrm{m}^{2}\right)$ de las cuencas visuales de los caminos de acceso al Paraje natural

Figura 27. Cuencas visuales de los caminos seleccionados y las torres vigia Figura 28. Propuesta de Itinerario y ubicación de miradores

Figura 29. Panorámica de los miradores

$>$ En primer lugar, se descartan los accesos que tienen una cuenca visual más limitada. Éstas se corresponden con los caminos 1, 3, $13,17,9,19$ y 18 respectivamente. El 1 y el 3, a pesar de que son dos de los tres únicos caminos que alcanzan la Torre de Maro, tienen unas cuencas visuales que quedan incluidas en el camino 2. La cuenca visual del camino 13 se restringe tan sólo al área de la Torre del Pino, estando dicha superficie comprendida en las visuales de otros muchos tramos representados $(2,4,5,6,8,10,12$, $14,15,16,20,21,22,23$ y 24). El resto de caminos enumerados arriba (el 17, 9, 19 y 18) se decide no incorporarlos al estudio porque sus áreas de visibilidad son muy reducidas y no se llegan a percibir ninguna de las torres.

> Siguiendo el orden de superficie (de menor a mayor) se destacan otro grupo de caminos $(7,4,6,5,15,11,14,8,12,2$ y 10), de los cuales se han elegido para el estudio el 6, 14, 2 y 10. Las cuencas visuales de los tramos 7, 4 y 5 aparecen contenidas en el área del camino 6, pero se ha preferido elegir este último porque desde ellos se visualizan un mayor número de torres (Torre del Río de la Miel, Torre del Pino, Torre Caleta y Cerro Gordo). El camino 15 se descarta por razones similares al caso anterior pero en este caso con respecto al camino 14. Sin embargo, en la elección de este último no se ha primado tanto el número de torres que alcanza, puesto que sólo comprende la ubicación de las Torre del Pino y Torre Caleta, como por ser la única cuenca visual que proyecta el área comprendida entre estas dos torres desde el límite del paraje por la carretera N-340 hasta la misma costa, recorriendo por completo una de las calas más pronunciadas del entorno. También se descartan los caminos 11,8 y 12 por quedar incluidas sus cuencas visuales en la superficie del camino 10, la cual incluye también 4 de las cinco torres.

> Los tramos que mayor superficie alcanzan en la zona del paraje, de mayor a menor, son los siguientes: 21, 23, 16, 22, 20 y 24. De estos trayectos se eligen para el estudio los caminos 21 y 16. El primero, porque tiene una cuenca visual espaciosa cuya distribución se extiende a lo largo de 4 torres (todas a excepción de la de Maro) y además, porque incluye las superficies de los campos de visión de los caminos 23, 22, 20 y 24. Por esta misma razón se decide prescindir de estos últimos recorridos. Por otra parte, se selecciona el camino 16, por incluir las superficies de las cuencas visuales de los caminos 14 y 21, representando transversalmente la zona de acceso a Torre Caleta y recorriendo su superficie la zona costera de dicha torre hacia el entorno de la Torre de Cerro-Gordo.

En definitiva, se puede observar que las vias que proporcionan áreas de mayor interés desde el punto de vista del paisaje son aquellas que se corresponden prácticamente con los tramos de acceso directo a las torres y su dominio más inmediato, lo que hace necesario establecer medidas de actuación necesarias para mantener estos elementos y sus condiciones de tránsito en buen estado. 
Elegidos los caminos de acceso más característicos, se procede a superponer las cuencas visuales de éstos a la de las torres para determinar cuales son las zonas comunes a todas ellas, y saber de este modo que áreas del paisaje dominan la visión desde las torres y desde sus vías de entrada desde el límite exterior del paraje hasta la línea de costa, sabiendo que las superficies incluidas dentro de estas áreas son susceptibles de ser observadas desde estos elementos, al igual que se conoce que aquellas zonas o elementos situados en el exterior de estas visuales no serán advertidos ${ }^{1}$.

En la figura 27 se puede apreciar que las cuencas visuales de las torres quedan totalmente inmersas en las de los caminos seleccionados, garantizándose desde éstos una adecuada visión de las mismas, y de forma reciproca, desde las áreas de visión de las torres se observan la totalidad de caminos elegidos y su entorno de observación, justificándose con ello la necesidad comentada anteriormente de establecer medidas de protección y valoración de los elementos de estas superficies claramente delimitadas.

A partir de estos resultados se propone el trazado de un itinerario de interés paisajístico que recorre de un extremo a otro el paraje, teniendo en cuenta las peculiaridades de los valores naturales y culturales que le imprimen personalidad. Para ello, se añaden a los mapas anteriores las coberturas correspondientes a las vías de comunicación principales, la red hidrográfica y los núcleos de población, extraídos del MVA 10.000, además de la ortofoto a color de 1999, que se interpretará visualmente para obtener información sobre la cobertura vegetal del suelo. El itinerario ira acompañado también de una propuesta de miradores con fines de observación para el uso y disfrute del patrimonio natural y cultural.

El itinerario propuesto (que puede recorrerse total o parcialmente) se ha realizado en función de los caminos actualmente transitables, aunque en algunos tramos sería quizás conveniente acortar el recorrido mediante el acondicionamiento de nuevos tramos (figura 28).

Los miradores propuestos, se sitúan entre la Torre de Maro y la Torre del Río de la Miel y en las planicies de la Torre del Pino y la Torre de Cerro Gordo. Estas localizaciones se han seleccionado por cubrir un radio de visibilidad bastante amplio, y por percibirse desde ellas las cinco torres que componen el patrimonio histórico del paraje. Se ubican en zonas llanas y elevadas próximas al mar, desde las que se pueden observar también el perfil topográfico del paisaje y las numerosas calas que salpican visualmente la costa (figura 29).

\section{Conclusiones}

Los territorios y paisajes andaluces son producto de la interacción de los grupos humanos y de estos con el medio físico a lo largo de toda su historia y, en este contexto, el Patrimonio Histórico es la huella de esa interacción y proporciona información acerca de cómo la socie-

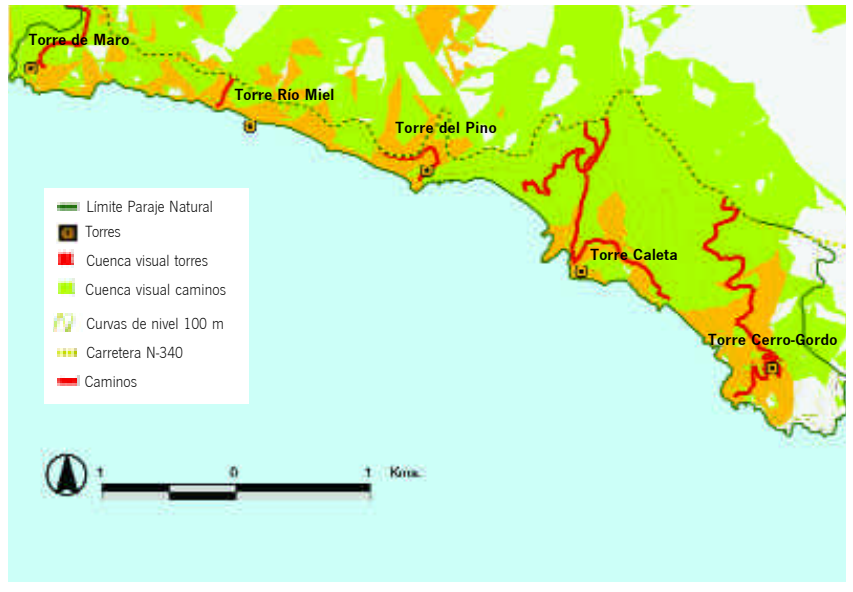

Figura 27

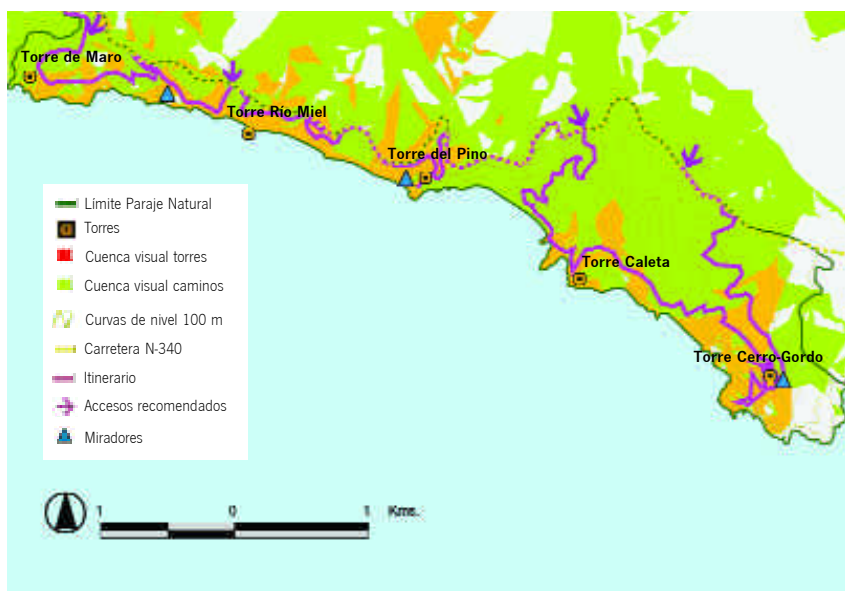

Figura 28

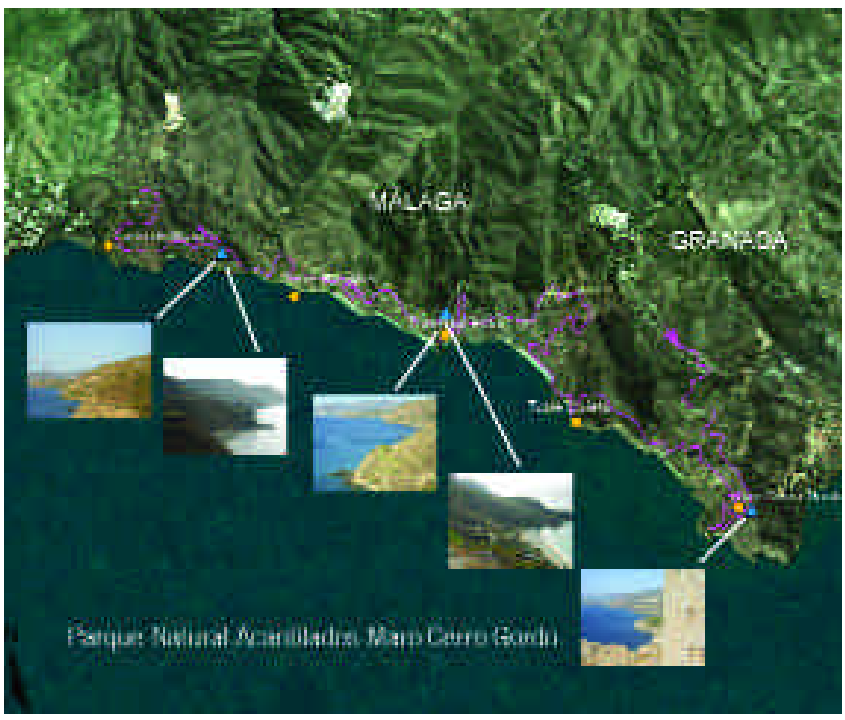

Figura 29 


\section{Criterios}

Torres de vigilancia costera y Paisaje:

integración de valores

culturales y naturales en

el Paraje Natural de

Maro-Cerro Gordo

\section{Marta Chiclana Rodríguez \\ Silvia Fernández Cacho}

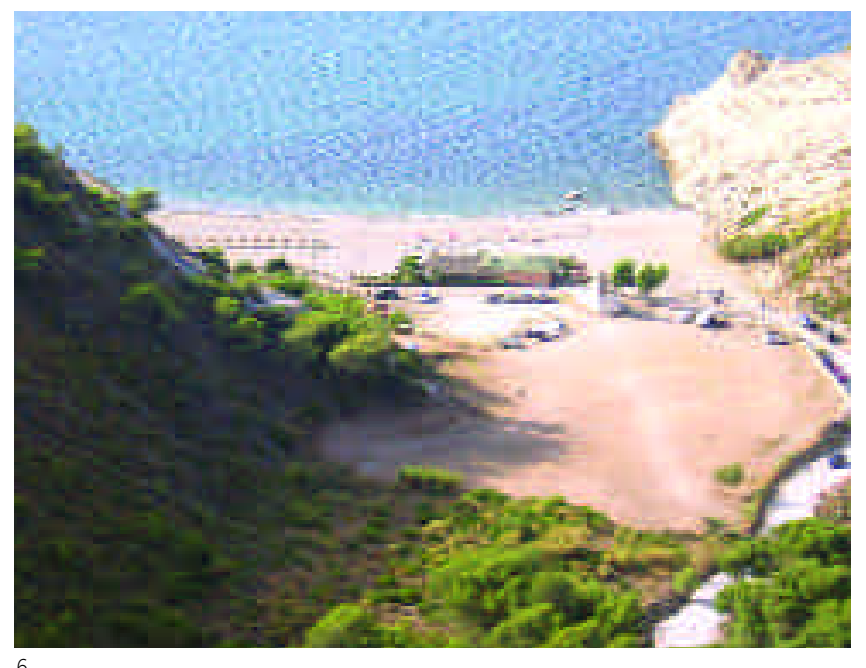

6

dad ha mantenido esa relación dialéctica con su entorno natural. Además, los propios vestigios arqueológicos se integran en la actualidad en el medio ambiente en el que se desarrollan las actividades de los seres humanos, de forma que espacios naturales que se han protegido por su singularidad perderian parte de su valor si aquellos no estuvieran presentes.

El objetivo de cualquier política territorial de promover actividades que entren en sinergia, es decir, que el efecto que provoque su acción combinada sea mayor que el resultante de la suma de las mismas actuando de forma independiente, debe tener en estos espacios un carácter singular en relación con la conservación y con el uso y disfrute de los recursos culturales y naturales (Martí, 2002).

Un sencillo análisis como el presentado en este trabajo, permite fundamentar propuestas que deben ser contrastadas sobre el terreno, en las que la oferta territorial de un espacio protegido aúne criterios naturales y culturales. Del mismo modo, puede ayudar a tomar decisiones relacionadas con los entornos de protección de los bienes culturales, entornos que son especialmente significativos en el caso de las torres vigía, a veces ubicadas en contextos que en la actualidad están fuertemente antropizados.

Precisamente la moderación y restricciones en los usos y aprovechamientos territoriales en los espacios naturales protegidos, permiten un mayor control de los impactos derivados de actuaciones territoriales especialmente agresivas con el medio. En el caso del Paraje Natural de Maro-Cerro Gordo, la protección ambiental ha incidido en la preservación de sus valores naturales, aunque en el entorno inmediato se están desarrollando actuaciones urbanísticas y prácticas de agricultura intensiva bajo plástico que afectan a la contemplación de sus recursos culturales y paisajísticos.
6. Imagen otoñal de la Playa de Cantarriján (entre Torre Caleta y Torre de Cerro Gordo)

7. Cultivos agrícolas bajo plástico. Al fondo, Torre Maro

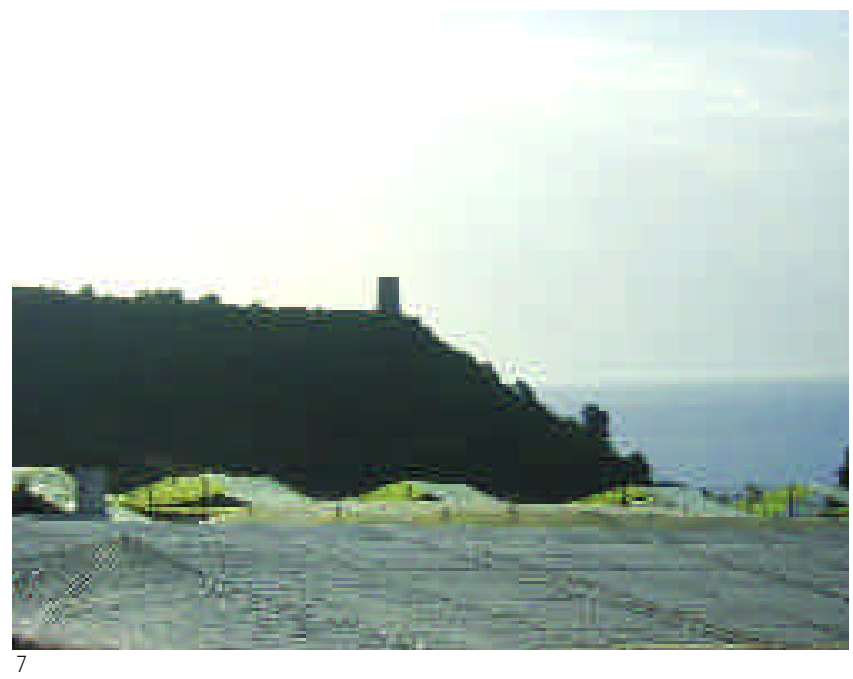

Para mejorar y preservar los valores naturales y culturales del Paraje se proponen adicionalmente las siguientes actuaciones:

> La mejora de las infraestructuras físicas, como garantía para regular la entrada y tránsito de los visitantes al interior del paraje. La afluencia de turistas en los últimos años está provocando una gran presión en el medio. La entrada con automóviles (imagen 6) y el desarrollo de conductas poco respetuosas con el medio ambiente (arrojo de basuras, arranque de vegetación, pintadas, ruidos...) generan un impacto negativo afectando directamente a la fragilidad del paisaje.

> La cercanía del paraje a la carretera N-340 está provocando precisamente, por la falta de acondicionamiento y control, la presencia de numerosas zonas afectadas por la acumulación de escombros y vertidos incontrolados (imagen 8). Asimismo, las zonas circundantes, ocupadas por los plásticos de los cultivos agrícolas intensivos están produciendo numerosos residuos, generando un evidente impacto visual (imagen 7), por lo que habria que reforzar el control sobre estos procesos.

$>$ Es necesario acondicionar las altiplanicies situadas en las inmediaciones de las torres con intervenciones mínimas que permitan aprovechar estos espacios a modo de miradores para la contemplación del paisaje (imagen 9).

Sirviéndose de esta circunstancia, se plantea ubicar en estos emplazamientos una serie de paneles interpretativos en los que se expliquen las peculiaridades de sus elementos de mayor interés y se analicen y representen los valores naturales y culturales más relevantes del ámbito del espacio protegido a fin de propiciar una conducta responsable y solidaria con el medio a través del conocimiento de sus cualidades, fomentando la conciencia y el compromiso social para su preservación. 


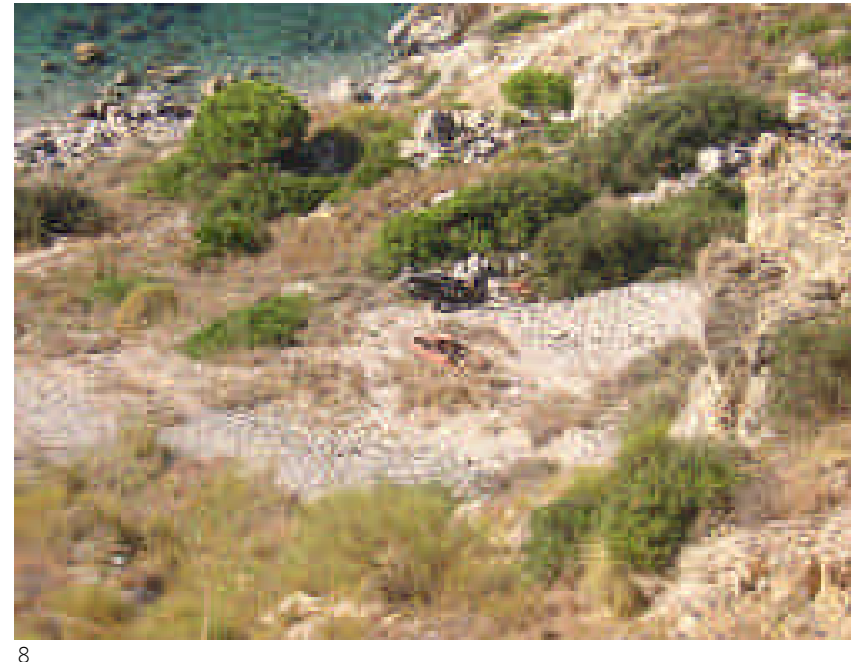

\section{Bibliografía}

BLANCKAERT, C. (1998) "La naturalisation de l'homme de Linné à Darwin. Archéologie du débat nature/culture" En DUCROS, A; DUCROS, J; JOULIAN, F. (Dir.) La culture est-elle naturelle? Editions Errance. Paris, 1998, pp. 15-24

BOUCHÉ, N. (1995) "Patrimoine culturel, patrimoine naturel en Europe: reflexions sur diverses expériences nationales et sur les collaborations internationales". Patrimoine culturel, patrimoine naturel. Colloque 12-13 décembre, 1994. La Documentation Française. París, 1995, pp. 192-212

CASTRO HENRIQUES, P. (2000) "Perspectivas na gestao de áreas protegidas em Portugal - o natural e cultural". O Verde. Patrimonio Natural e Cultural de un olghar comun a una intervençao integrada. GEOTA. Caldas da Rainha, 2000, pp. 39-51

CONSEJERÍA DE MEDIO AMBIENTE (2003) Itinerarios del Paraje Natural Acantilados de Maro-Cerro Gordo. Consejeria de Medio Ambiente. Junta de Andalucia, 2003

CORBEY, R. (1998) "De l'histoire naturelle a l'histoire humaine: comment conceptualiser les origins de la culture?" En DUCROS, A; DUCROS, J; JOULIAN, F .(Dir.): La culture est-elle naturelle?. Editions Errance. Paris, 1998, pp. 223-238

DIRECTIVA 92/43/CEE del Consejo, de 21 de mayor, relativa a la Conservación de los Hábitats Naturales y de la flora y fauna silvestres (DOCE L 206/1992, de 22 de julio)

GESTIN, J.P. (1995) "Le parc naturel régional d'Armorique". Patrimoine culturel, patrimoine naturel. Colloque 12-13 décembre, 1994. La Documentation Française. Paris, 1995, pp. 94-101

GUTIERREZ-CORTINES CORRAL, C. (2002) "Desarrollo sostenible y el Patrimonio Cultural y Natural". GUTIERREZ-CORTINES CORRAL, C. (Ed.) Desarrollo sostenible y patrimonio histórico y natural. Una nueva mirada hacia la renovación del pasado. Fundación Marcelino Botín. Santander, 2002, pp. 29-115

KUPER, A. (1998) "Les origines de l'idée moderne de culture en Anthropologie". DUCROS, A; DUCROS, J; JOULIAN, F. (Dir.) La culture est-elle naturelle? Editions Errance. Paris, 1998, pp. 55-69

LEY 2/89, de 18 de julio, por el que se aprueba el inventario de Espacios Naturales

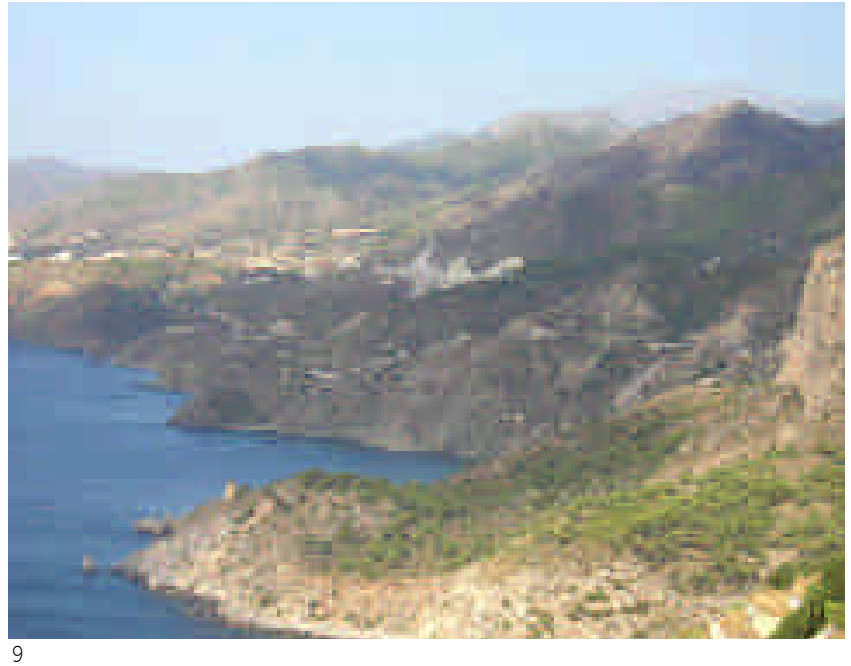

Protegidos de Andalucia y se establecen medidas adicionales para su protección (BOJA 60/1989, de 27 de julio; BOE 201/1989, de 23 de agosto)

MARTí REGUÉ, X. (2002) "Impacte ambiental i patrimoni cultural". II Jornada sobre La Gestió del Patrimoni Sostenible: Patrimoni Cultural i Medi Ambien. Castell de Castellet. 15 de novembre de 2002. En: www.fundacioabertis.org/ cat/html/jornades/jornada_gestio_patrimoni_sostenible.doc [consultado el 10 de agosto de 2004]

NUZzo, A. (1999) "Parchi naturali/parchi culturali: un'integrazione necessaria". En LENZI, F. (a cura di) Archeologia e Ambiente. Atti del convegno internacionale. FerraraFiere 3-4 aprile, 1998. Documenti/30. Istituto per i beni artistici, culturali e naturali della regione Emilia-Romagna. Forli, 1999, pp.329-332

PEANO, A. (1999) "Parchi naturali e parchi culturali: un'integrazione possibile" En FRANCOVICH, R. y ZIFFERERO, A. (a cura di): Musei e Parchi Archeologici. IX ciclo di lezioni sulla ricerca applicata in Archeologia. Certosa di Pontignano (Siena) dicembre, 1997, 15-21. Universitá di Siena. CNRS. Firenze, 1999, pp. 183-189

PROTOCOLO sobre las zonas especialmente protegidas y la diversidad biológica en el Mediterráneo y anexos, adoptado en Barcelona el 10 de junio de 1995 y en Montecarlo el 24 de noviembre de 1996, respectivamente (DOCE L322/1999, de 14 de diciembre)

ROSMANIUHO, L. (2000) "Introduçao ao tema". O Verde. Patrimonio Natural e Cultural de un olghar comun a una intervençao integrada. GEOTA. Caldas da Rainha, 2000, pp. 7-11

VAN DER LEEUW, S. (1998) "La nature serait'elle d'origine culturelle? Histoire, Archéologie, Sciences Naturelles et Environnement". En DUCROS, A; DUCROS, J; JOULIAN, F. (Dir.) La culture est-elle naturelle? Editions Errance. Paris, 1998, pp. 83-98

ZIFFERERO, A. (1999) "Archeologia e ambiente: note sulla situazione italiana, tra necessità di conservazione e prospettive di ricerca". En LENZI, F. (a cura di) Archeologia e Ambiente. Atti del convegno internacionale. FerraraFiere 3-4 aprile, 1998. Documenti/30. Istituto per i beni artistici, culturali e naturali della regione EmiliaRomagna. Forli, 1999, pp. 319-328

Notas

${ }^{1}$ Por ejemplo, el vacio que se observa en el sector más suroriental del paraje se explica por la disposición altitudinal de la orografía local, no incluyéndose dicho sector en ninguna de las cuencas visuales de los caminos ni de las torres vigia. 